\title{
A prospective observational study of early intervention with erythropoietin therapy and renal survival in non-dialysis chronic kidney disease patients with anemia: JET-STREAM Study
}

\author{
Tadao Akizawa $^{1}$ - Yoshiharu Tsubakihara ${ }^{2}$ Hideki Hirakata ${ }^{3}$ - Yuzo Watanabe ${ }^{4}$. \\ Hiroki Hase $^{5} \cdot$ Shinichi Nishi $^{6} \cdot$ Tetsuya Babazono $^{7} \cdot$ Michiko Kumagai $^{8}$. \\ Shingo Katakura ${ }^{8}$ - Yukari Uemura $^{9} \cdot$ Yasuo Ohashi $^{10} \cdot$ JET-STREAM Study Group $^{2}$
}

Received: 14 September 2015/Accepted: 27 December 2015/Published online: 16 January 2016

(c) The Author(s) 2016. This article is published with open access at Springerlink.com

\begin{abstract}
Background There is limited data showing that early treatment for anemia could prolong renal survival in nondialysis chronic kidney disease (CKD) patients. We therefore investigated the relationship between hemoglobin (Hb) levels at initiation of epoetin beta therapy and renal outcome in non-dialysis CKD patients with anemia.

Methods In this prospective, multi-center, observational study, non-dialysis CKD patients with anemia who were naïve to erythropoiesis-stimulating agents (ESAs) were divided into three groups based on their $\mathrm{Hb}$ levels at initiation of epoetin beta therapy (Group I: $10 \leq \mathrm{Hb}<11 \mathrm{~g}$ / dL, Group II: $9 \leq \mathrm{Hb}<10 \mathrm{~g} / \mathrm{dL}$, and Group III: $\mathrm{Hb}<9 \mathrm{~g} / \mathrm{dL}$ ). The primary endpoint was time to first occurrence of any renal event. For the primary analysis, an inverse probability weighted Cox regression model was
\end{abstract}

The members of JET-STREAM Study Group are listed under the Acknowledgments.

Michiko Kumagai

ishikawamck@chugai-pharm.co.jp

1 Division of Nephrology, Department of Medicine, Showa University School of Medicine, 1-5-8 Hatanodai, Shinagawa-ku, Tokyo 142-8666, Japan

2 Course of Safety Management in Health Care Sciences, Graduate School of Health Care Sciences, Jikei Institute, Osaka, Japan

3 Division of Nephrology and Dialysis Center, Japanese Red Cross Fukuoka Hospital, Fukuoka, Japan

4 Department of Internal Medicine, Kasugai Municipal Hospital, Nagoya, Japan

5 Department of Nephrology, Toho University Ohashi Medical Center, Tokyo, Japan used to adjust time-dependent selection bias in the artificially censored data.

Results A total of 1113 patients were eligible for primary endpoint analysis. Risk of renal events was significantly higher in Group III compared with Group I (HR, 2.52; $95 \%$ CI, 1.98-3.21; $P<0.0001)$; although not significant, the risk was also higher in Group II compared with Group I (HR, 1.48; $95 \%$ CI, 0.91-2.40; $P=0.11$ ).

Conclusion Initiation of ESA therapy when $\mathrm{Hb}$ levels decreased below $11 \mathrm{~g} / \mathrm{dL}$ but not below $10 \mathrm{~g} / \mathrm{dL}$ could be more effective at reducing the risk of renal events in nondialysis CKD patients with anemia compared with initiation of ESA therapy at below $9 \mathrm{~g} / \mathrm{dL}$ or even $10 \mathrm{~g} / \mathrm{dL}$.

Keywords Anemia - Chronic kidney disease . Erythropoiesis-stimulating agents $\cdot$ Non-dialysis $\cdot$ Renal survival

6 Division of Nephrology and Kidney Center, Kobe University Graduate School of Medicine, Kobe, Japan

7 Division of Nephrology and Hypertension, Diabetes Center, Tokyo Women's Medical University School of Medicine, Tokyo, Japan

8 Pharmacovigilance Department, Chugai Pharmaceutical Co. Ltd., 2-1-1 Nihonbashi-Muromachi, Chuo-ku, Tokyo 103-8324, Japan

9 Biostatistics Division, Clinical Research Support Center, The University of Tokyo Hospital, Tokyo, Japan

10 Department of Integrated Science and Engineering for Sustainable Society, Chuo University, Tokyo, Japan 


\section{Introduction}

Anemia is a common complication in patients with chronic kidney disease (CKD) and is primarily caused by declining erythropoietin production in such patients [1]. Anemia can worsen renal and cardiac function and is associated with an increased risk of mortality or hospitalization [2]. Thus, treatment of anemia in CKD patients is especially important. Erythropoiesis-stimulating agents (ESAs) have been used for the treatment of anemia in such patients. Correction of anemia with ESAs is associated with improved outcome [3] and quality of life [4].

There have been many reports of ESA studies, and some have discussed appropriate target hemoglobin $(\mathrm{Hb})$ levels for maintenance with ESAs. The CHOIR study failed to show the benefit of setting a high target $\mathrm{Hb}$ level and suggested a potential for increased composite risk of death and cardiovascular events [5]. The TREAT study also showed that setting a high target $\mathrm{Hb}$ level provided no clinical benefit and, instead, increased the risk of cerebrovascular disease [6]. The appropriate target $\mathrm{Hb}$ level for ESA therapy remains controversial.

Still, there have been few reports on an appropriate $\mathrm{Hb}$ level for starting ESA therapy. Although the 2012 guideline from Kidney Disease: Improving Global Outcomes states that introduction of ESA therapy should be considered when $\mathrm{Hb}$ level decreases below $10 \mathrm{~g} / \mathrm{dL}$ in patients with non-dialysis-dependent CKD [7], it provides no evidence to support this recommendation. Some evidence is provided by Gouva et al., who found that early intervention with ESAs in anemia slows the progression of renal disease and delays the initiation of renal replacement therapy [8]. Although early detection and management of anemia is considered to be vital, the best timing for starting ESA therapy is still uncertain, and it is now imperative that we collect data on the appropriate $\mathrm{Hb}$ level for starting ESA therapy.

The JET-STREAM (Japan Erythropoietin Treatment survey for STarting hemoglobin level in REnal Anemia Management) study was conducted to investigate the relationship between renal outcome and the $\mathrm{Hb}$ level at initiation of epoetin beta therapy, rather than the target $\mathrm{Hb}$ level, in non-dialysis CKD patients with anemia.

\section{Methods}

\section{Study population}

Patients were recruited from February 2010 to March 2011. Eligible patients were non-dialysis, ESA-naïve, CKD patients with anemia who were scheduled to start epoetin beta therapy. Patients were not scheduled for renal replacement therapy within at least the following 6 months. Patients with non-renal anemia or with an estimated glomerular filtration rate (eGFR) [9] of less than $6 \mathrm{~mL} /$ $\min / 1.73 \mathrm{~m}^{2}$ were excluded. All study participants provided written informed consent.

\section{Study design and measurements}

This study was a prospective, observational study. Epoetin beta (EPOGIN 1500, 3000, 6000, 9000 and 12,000 IU, Chugai Pharmaceutical, Tokyo, Japan) was used for this study according to the package insert approved by the Ministry of Health, Labour and Welfare in Japan [10]. Patients were followed up for a maximum of 2 years from initiation of epoetin beta therapy or until discontinuation of therapy, initiation of renal replacement therapy, death, malignancy, withdrawal of consent, or loss of follow-up.

Information was collected on patient baseline characteristics (age, sex, medical history, comorbidities), epoetin beta treatment status, treatment status of any other drugs, inpatient/outpatient status, the date renal replacement therapy was introduced, laboratory test values, and adverse reactions. Data on $\mathrm{Hb}$ and serum creatinine ( $\mathrm{sCr}$ ) levels were collected not only during the epoetin beta treatment period but also retrospectively on the dates that $\mathrm{Hb}$ levels decreased below $11 \mathrm{~g} / \mathrm{dL}$ for the first time.

The study protocol was approved by each local institutional review board (approval no. at Osaka General Medical Center: 21-562), and the study was conducted in accordance with the Declaration of Helsinki and Japanese Ministry of Health, Labour and Welfare regulations for postmarketing surveillance. This study is registered with the University Hospital Medical Information Network (ID: UMIN000003116).

The primary endpoint was time to first occurrence of any renal event (defined as initiation of renal replacement therapy, doubling of sCr level, or measurement of eGFR less than $6.0 \mathrm{~mL} / \mathrm{min} / 1.73 \mathrm{~m}^{2}$ ). Secondary endpoints were occurrence of cardiovascular events (defined as death or hospitalization from heart failure, angina, myocardial infarction, cerebral infarction, intracranial cerebral hemorrhage, or transient ischemic attack) and safety. Relationships between patient baseline characteristics and outcomes were also evaluated.

\section{Statistical analysis}

Eligible patients were divided into the following three groups based on their $\mathrm{Hb}$ levels at initiation of epoetin beta therapy: Group I consisted of patients with $10 \leq \mathrm{Hb}<11 \mathrm{~g} / \mathrm{dL}$, Group II with $9 \leq \mathrm{Hb}<10 \mathrm{~g} / \mathrm{dL}$, and Group III with $\mathrm{Hb}<9 \mathrm{~g} / \mathrm{dL}$. If the time of epoetin beta 
therapy initiation was defined as the starting point of survival analysis, later starters may be at a disadvantage because of the lead time from the earlier stage to therapy initiation (i.e., their $\mathrm{Hb}$ levels may be higher) and because of their relatively poor physical condition compared with earlier starters. To account for this lead-time bias, the primary endpoint was analyzed from the day $\mathrm{Hb}$ levels decreased below $11 \mathrm{~g} / \mathrm{dL}$ for the first time (Fig. 1), and a recently developed statistical concept called "dynamic treatment regime" [11] was applied for group comparisons.

For comparison of the three groups, we analyzed Group I vs Group II and Group I vs Group III. The treatment strategy for each group was dynamic in that the decision to initiate treatment was guided by each patient's developing clinical status (in this study, Hb level). Hernan et al. [11] have shown that the relative efficacy of a dynamic treatment regime can be evaluated and compared using inverse probability weighting (IPW), which was proposed by Robins et al. [12]. To compare two dynamic treatment regimes, we artificially censored those patients who deviated from one of the two regimes of interest; however, uncensored patients may have different risk factor profiles from censored patients. We used the IPW Cox regression model to adjust for this time-dependent selection bias in the artificially censored patients. We estimated each patient-specific weight using the inverse of each patient's estimated probability of remaining uncensored. These probabilities were estimated by fitting a pooled logistic regression model to the conditional probability of the remaining uncensored group at each visit given the history of covariates. The covariates in the model included baseline- and time-dependent risk factors for renal and cardiovascular events: Hb levels, sCr levels, age, sex, and comorbidities observed in more than $5 \%$ of patients in each group (i.e., hypertension, heart failure, angina, arrhythmia, diabetes, hyperlipidemia, and hyperuricemia). To increase precision in estimation, we used a stabilized weight with the numerator representing the probability of each patient remaining uncensored given only the baseline covariates and the denominator with time-dependent covariates [13]. Data missing from continuous baseline variables were substituted by the baseline mean value of all patients.

The Cox regression model was used to assess relationships between patient baseline characteristics (including Hb levels, sCr levels, age, sex, comorbidities, and medical history; comorbidities and medical histories observed in more than $5 \%$ of patients in each group and with less than $20 \%$ of data missing were included) and outcomes for those patients eligible for efficacy analysis. Values of $P<0.05$ were considered statistically significant. All analyses were conducted using SAS v9.3 (SAS Institute, Cary, NC, USA).

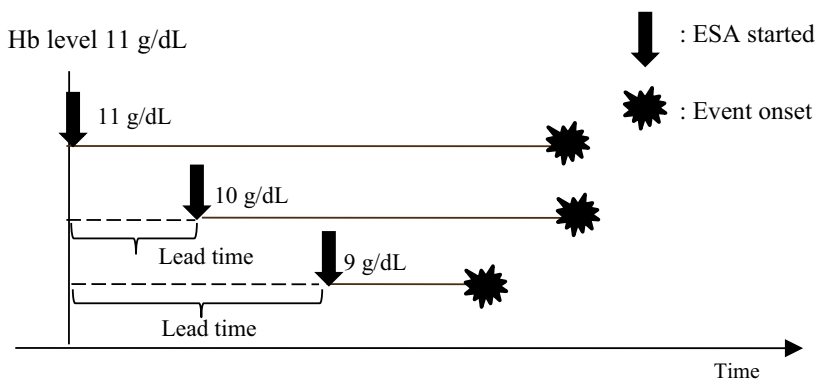

Fig. 1 Study design accounting for lead-time bias. To account for lead-time bias, the date, $\mathrm{Hb}$ levels, and $\mathrm{sCr}$ levels were confirmed at the time $\mathrm{Hb}$ levels decreased below $11 \mathrm{~g} / \mathrm{dL}$ for the first time. Analysis was started from the point $\mathrm{Hb}$ levels decreased below $11 \mathrm{~g} /$ $\mathrm{dL}$

\section{Results}

\section{Patients and baseline characteristics}

Of 1826 patients screened, 112 patients were excluded because they withdrew consent or did not receive epoetin beta therapy, leaving 1714 patients eligible for safety analysis in this study. A total of 1645 patients were eligible for efficacy analysis, and 1113 patients were eligible for analysis of the primary endpoint (Fig. 2). Patients who were eligible for primary endpoint analysis were divided into 3 groups based on $\mathrm{Hb}$ levels at initiation of epoetin beta: Group I $(10 \leq \mathrm{Hb}<11 \mathrm{~g} / \mathrm{dL})$ had 309 patients, Group II ( $9 \leq \mathrm{Hb}<10 \mathrm{~g} / \mathrm{dL})$ had 545 patients, and Group III $(\mathrm{Hb}<9 \mathrm{~g} / \mathrm{dL})$ had 259 patients. The characteristics of these patients are summarized in Table 1. The mean eGFR levels on the day the $\mathrm{Hb}$ levels decreased below $11 \mathrm{~g} / \mathrm{dL}$ for the first time in Groups I, II, and III were $23.6 \pm 12.3 \mathrm{~mL} / \mathrm{min} / 1.73 \mathrm{~m}^{2}$ (median, $20.7 \mathrm{~mL} / \mathrm{min} /$ $1.73 \mathrm{~m}^{2}$ ), $24.6 \pm 13.1 \mathrm{~mL} / \mathrm{min} / 1.73 \mathrm{~m}^{2}$ (median, $21.8 \mathrm{~mL} /$ $\mathrm{min} / 1.73 \mathrm{~m}^{2}$ ), and $27.7 \pm 16.8 \mathrm{~mL} / \mathrm{min} / 1.73 \mathrm{~m}^{2}$ (median, $22.9 \mathrm{~mL} / \mathrm{min} / 1.73 \mathrm{~m}^{2}$ ), respectively. At this time, the respective $\mathrm{Hb}$ levels in Groups I, II, and III were $10.5 \pm 0.4 \mathrm{~g} / \mathrm{dL}, 10.5 \pm 0.5 \mathrm{~g} / \mathrm{dL}$, and $10.4 \pm 0.5 \mathrm{~g} / \mathrm{dL}$ (Table 2).

\section{Primary endpoint}

Renal events occurred in 100 patients (32.4\%) in Group I, 246 patients $(45.1 \%)$ in Group II, and 157 patients $(60.6 \%)$ in Group III

The calculated weights between Groups I and II and between Groups I and III are summarized in Table 3. When Groups I and II were compared, some patients had extreme weights. It is well known that such extreme weights result in a large mean square of the estimated effect. To account for these extreme weights, we truncated the weight at the 


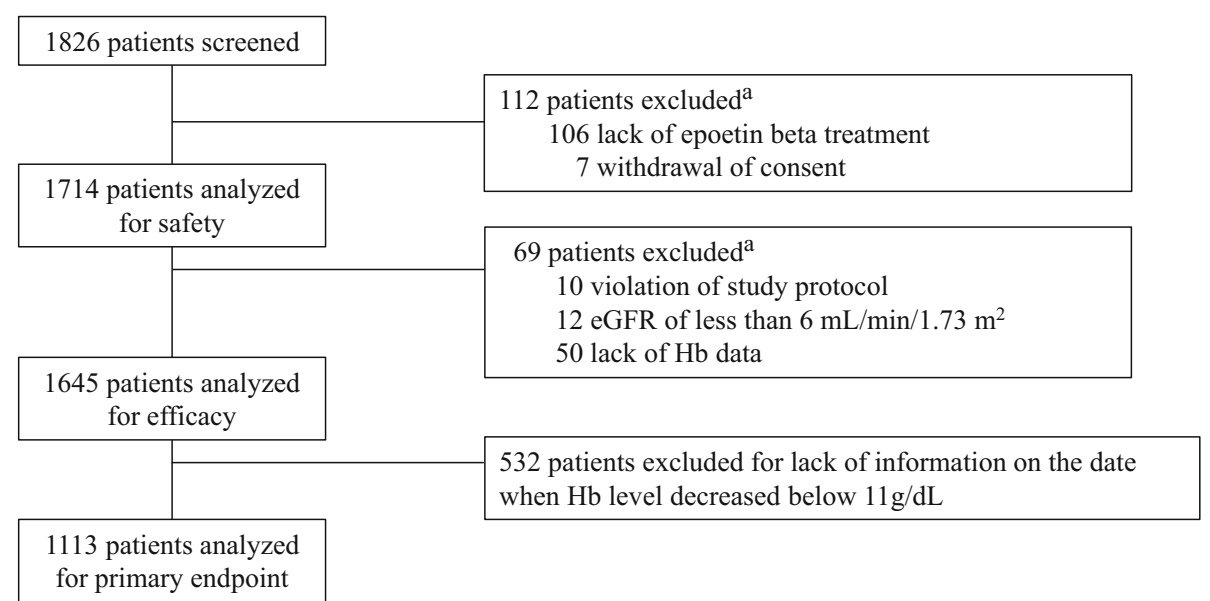

Fig. 2 Study profile. Of 1826 patients screened, 112 were excluded because they did not receive epoetin beta treatment or they withdrew consent. The remaining 1714 patients were eligible for safety analysis in this study. 1645 patients were eligible for efficacy analysis, and

99th percentile. That is, weights larger than the 99th percentile were set to the value of the 99th percentile. When Groups I and III were compared, the calculated weights were not as large, and truncation of the weights was unnecessary. After adjusting for artificial censoring using the IPW method, the risk of renal events in Group III was significantly higher than in Group I (HR, 2.52; $95 \%$ CI, $1.98-3.21 ; P<0.0001)$; and although not significant, this risk was higher in Group II than in Group I (HR, 1.48; $95 \%$ CI, 0.91-2.40; $P=0.11$ ) (Table 3A). For sensitivity analysis, we also used a weight truncated at the 98th percentile to compare Groups I and II. Risk of renal events in Group II was significantly higher than in Group I (HR, 1.29; $95 \%$ CI, 1.02-1.64; $P=0.033$ ) (Table 3B).

\section{Secondary endpoints}

Cardiovascular events occurred in 24 patients $(7.8 \%)$ in Group I, 52 patients $(9.5 \%)$ in Group II, and 31 patients $(12.0 \%)$ in Group III. Since the weights were extremely large for the comparison between Groups I and II, we truncated the weights at the 98th percentile. Based on the IPW method, the risk of cardiovascular events did not differ between Group I and Group II, and this risk in Group III was higher than in Group I, but not significantly (HR, 1.94; $95 \%$ CI, 0.96-3.94; $P=0.066$ ) (Table 4).

To evaluate the relationships between patient baseline characteristics and outcomes, we used data from 1645 patients. The main statistically significant clinical variables associated with poor renal survival were lower $\mathrm{Hb}$ levels, higher sCr levels, lower serum albumin levels, comorbid diabetes mellitus, and previous diuretic use (Fig. 3).
1113 patients with information on the date when $\mathrm{Hb}$ levels decreased below $11 \mathrm{~g} / \mathrm{dL}$ were eligible for analysis of the primary endpoint. ${ }^{\mathrm{a}}$ Numbers of excluded patients in this figure overlap

Of the 1714 patients in the safety analysis set, adverse reactions related to epoetin beta were reported in 13 patients $(0.8 \%)$ (Table 5). Of these, serious adverse reactions were reported in five patients $(0.3 \%)$ : cerebral hemorrhage in two patients and cerebral infarction, acute myocardial infarction, and aortic aneurysm rupture in one patient each.

\section{Discussion}

A randomized comparative study design would be preferable for exploring the appropriate timing (Hb level) for initiation of ESA therapy. However, such a design presents ethical difficulties given concerns that patients with depleted $\mathrm{Hb}$ levels might be deprived of an opportunity for anemia treatment. Therefore, an observational study design becomes the realistic choice, but such a design itself presents two issues that should be noted: lead-time bias and selection bias. And a valid assessment is impossible without first taking these biases into account.

With the first issue, lead-time bias, renal function in a group starting ESA treatment at a lower $\mathrm{Hb}$ level will be worse than that in a group starting ESA treatment at a higher $\mathrm{Hb}$ level. Therefore, the time to onset of events in the former group will be underestimated because it will appear shorter (Fig. 1). To eliminate such bias in this study, the date, $\mathrm{Hb}$ levels, and $\mathrm{sCr}$ levels were confirmed at the time $\mathrm{Hb}$ levels decreased below $11 \mathrm{~g} / \mathrm{dL}$ for the first time, and analysis used this data rather than the data from initiation of ESA treatment. Although there was variation in patient background characteristics such as sex, age, and 
Table 1 Characteristics of patients

\begin{tabular}{|c|c|c|c|c|c|c|}
\hline \multirow[t]{2}{*}{ Variables } & \multirow{2}{*}{$\begin{array}{l}\text { Patients analyzed } \\
\text { for efficacy } \\
(N=1645)\end{array}$} & \multirow{2}{*}{$\begin{array}{l}\text { Patients analyzed } \\
\text { for primary } \\
\text { endpoint } \\
(N=1113)\end{array}$} & \multicolumn{3}{|c|}{ Sub-groups based on $\mathrm{Hb}$ levels at initiation of epoetin beta } & \multirow[t]{2}{*}{$P$ value } \\
\hline & & & $\begin{array}{l}\text { Group I } \\
10 \leq \mathrm{Hb}<11 \\
\mathrm{~g} / \mathrm{dL}(N=309)\end{array}$ & $\begin{array}{l}\text { Group II } \\
9 \leq \mathrm{Hb}<10 \\
\mathrm{~g} / \mathrm{dL}(N=545)\end{array}$ & $\begin{array}{l}\text { Group III } \\
\mathrm{Hb}<9 \mathrm{~g} / \mathrm{dL} \\
(N=259)\end{array}$ & \\
\hline \multicolumn{7}{|l|}{ Sex } \\
\hline Male & $1039(63.2 \%)$ & $727(65.3 \%)$ & $222(71.8 \%)$ & $348(63.9 \%)$ & $157(60.6 \%)$ & 0.012 \\
\hline Age (years) ${ }^{\mathrm{a}}$ & $70.5 \pm 12.6$ & $70.5 \pm 12.4$ & $69.0 \pm 11.9$ & $70.7 \pm 12.8$ & $71.9 \pm 12.1$ & 0.021 \\
\hline \multicolumn{7}{|l|}{ Cause of CKD } \\
\hline $\begin{array}{l}\text { Chronic } \\
\text { glomerulonephritis }\end{array}$ & $345(21.0 \%)$ & $249(22.4 \%)$ & $64(20.7 \%)$ & $130(23.9 \%)$ & $55(21.2 \%)$ & 0.50 \\
\hline Diabetic nephropathy & $579(35.2 \%)$ & $388(34.9 \%)$ & $113(36.6 \%)$ & $179(32.8 \%)$ & $96(37.1 \%)$ & 0.38 \\
\hline Nephrosclerosis & $391(23.8 \%)$ & $268(24.1 \%)$ & $80(25.9 \%)$ & $129(23.7 \%)$ & $59(22.8 \%)$ & 0.66 \\
\hline \multicolumn{7}{|l|}{ Medical history } \\
\hline Heart failure & $91(5.5 \%)$ & $58(5.2 \%)$ & $18(5.8 \%)$ & $25(4.6 \%)$ & $15(5.8 \%)$ & 0.66 \\
\hline Angina & $92(5.6 \%)$ & $67(6.0 \%)$ & $19(6.1 \%)$ & $30(5.5 \%)$ & $18(6.9 \%)$ & 0.72 \\
\hline Arrhythmia & $54(3.3 \%)$ & $42(3.8 \%)$ & $9(2.9 \%)$ & $15(2.8 \%)$ & $18(6.9 \%)$ & 0.0091 \\
\hline Myocardial infarction & $83(5.0 \%)$ & $55(4.9 \%)$ & $12(3.9 \%)$ & $26(4.8 \%)$ & $17(6.6 \%)$ & 0.33 \\
\hline $\begin{array}{l}\text { Peripheral arterial } \\
\text { disease }\end{array}$ & $20(1.2 \%)$ & $15(1.3 \%)$ & $4(1.3 \%)$ & $7(1.3 \%)$ & $4(1.5 \%)$ & 0.95 \\
\hline Cerebral infarction & $181(11.0 \%)$ & $130(11.7 \%)$ & $36(11.7 \%)$ & $63(11.6 \%)$ & $31(12.0 \%)$ & 0.99 \\
\hline Cerebral hemorrhage & $39(2.4 \%)$ & $28(2.5 \%)$ & $7(2.3 \%)$ & $14(2.6 \%)$ & $7(2.7 \%)$ & 0.94 \\
\hline Malignancy & $216(13.1 \%)$ & $149(13.4 \%)$ & $41(13.3 \%)$ & $69(12.7 \%)$ & $39(15.1 \%)$ & 0.65 \\
\hline \multicolumn{7}{|l|}{ Complications } \\
\hline Hypertension & $1353(82.2 \%)$ & $919(82.6 \%)$ & $246(79.6 \%)$ & $462(84.8 \%)$ & $211(81.5 \%)$ & 0.14 \\
\hline Heart failure & $149(9.1 \%)$ & $87(7.8 \%)$ & $24(7.8 \%)$ & $32(5.9 \%)$ & $31(12.0 \%)$ & 0.011 \\
\hline Cardiac hypertrophy & $56(3.4 \%)$ & $30(2.7 \%)$ & $9(2.9 \%)$ & $15(2.8 \%)$ & $6(2.3 \%)$ & 0.90 \\
\hline Angina & $129(7.8 \%)$ & $94(8.4 \%)$ & $23(7.4 \%)$ & $49(9.0 \%)$ & $22(8.5 \%)$ & 0.74 \\
\hline Arrhythmia & $100(6.1 \%)$ & $69(6.2 \%)$ & $18(5.8 \%)$ & $34(6.2 \%)$ & $17(6.6 \%)$ & 0.93 \\
\hline Myocardial infarction & $38(2.3 \%)$ & $26(2.3 \%)$ & $8(2.6 \%)$ & $12(2.2 \%)$ & $6(2.3 \%)$ & 0.94 \\
\hline $\begin{array}{l}\text { Peripheral arterial } \\
\text { disease }\end{array}$ & $48(2.9 \%)$ & $34(3.1 \%)$ & $14(4.5 \%)$ & $13(2.4 \%)$ & $7(2.7 \%)$ & 0.20 \\
\hline Cerebral infarction & $73(4.4 \%)$ & $46(4.1 \%)$ & $16(5.2 \%)$ & $16(2.9 \%)$ & $14(5.4 \%)$ & 0.14 \\
\hline Cerebral hemorrhage & $3(0.2 \%)$ & $3(0.3 \%)$ & $1(0.3 \%)$ & $2(0.4 \%)$ & $0(0.0 \%)$ & 0.81 \\
\hline $\begin{array}{l}\text { Transient ischemic } \\
\text { attack }\end{array}$ & $7(0.4 \%)$ & $6(0.5 \%)$ & $2(0.6 \%)$ & $2(0.4 \%)$ & $2(0.8 \%)$ & 0.77 \\
\hline Diabetes mellitus & $734(44.6 \%)$ & $495(44.5 \%)$ & $145(46.9 \%)$ & $228(41.8 \%)$ & $122(47.1 \%)$ & 0.22 \\
\hline Diabetic retinopathy & $244(14.8 \%)$ & $167(15.0 \%)$ & $43(13.9 \%)$ & $81(14.9 \%)$ & $43(16.6 \%)$ & 0.67 \\
\hline Hyperlipidemia & $499(30.3 \%)$ & $369(33.2 \%)$ & $111(35.9 \%)$ & $185(33.9 \%)$ & $73(28.2 \%)$ & 0.13 \\
\hline Hepatitis B & $11(0.7 \%)$ & $7(0.6 \%)$ & $2(0.6 \%)$ & $4(0.7 \%)$ & $1(0.4 \%)$ & 0.90 \\
\hline Hepatitis C & $56(3.4 \%)$ & $39(3.5 \%)$ & $10(3.2 \%)$ & $21(3.9 \%)$ & $8(3.1 \%)$ & 0.82 \\
\hline Gastrointestinal ulcer & $62(3.8 \%)$ & $36(3.2 \%)$ & $10(3.2 \%)$ & $13(2.4 \%)$ & $13(5.0 \%)$ & 0.14 \\
\hline $\begin{array}{l}\text { Secondary } \\
\text { hyperparathyroidism }\end{array}$ & $62(3.8 \%)$ & $42(3.8 \%)$ & $11(3.6 \%)$ & $22(4.0 \%)$ & $9(3.5 \%)$ & 0.90 \\
\hline Hyperphosphatemia & $47(2.9 \%)$ & $29(2.6 \%)$ & $4(1.3 \%)$ & $15(2.8 \%)$ & $10(3.9 \%)$ & 0.15 \\
\hline Hyperuricemia & $503(30.6 \%)$ & $363(32.6 \%)$ & $96(31.1 \%)$ & $176(32.3 \%)$ & $91(35.1 \%)$ & 0.57 \\
\hline History of smoking & $445(27.1 \%)$ & $313(28.1 \%)$ & $87(28.2 \%)$ & $155(28.4 \%)$ & $71(27.4 \%)$ & 0.96 \\
\hline $\begin{array}{l}\text { History of } \\
\text { hospitalization }\end{array}$ & $911(55.4 \%)$ & $665(59.7 \%)$ & $161(52.1 \%)$ & $335(61.5 \%)$ & $169(65.3 \%)$ & 0.033 \\
\hline $\begin{array}{l}\text { History of blood } \\
\text { transfusion }\end{array}$ & $103(6.3 \%)$ & $68(6.1 \%)$ & $11(3.6 \%)$ & $36(6.6 \%)$ & $21(8.1 \%)$ & 0.097 \\
\hline
\end{tabular}


Table 1 continued

\begin{tabular}{|c|c|c|c|c|c|c|}
\hline \multirow[t]{2}{*}{ Variables } & \multirow{2}{*}{$\begin{array}{l}\text { Patients analyzed } \\
\text { for efficacy } \\
(N=1645)\end{array}$} & \multirow{2}{*}{$\begin{array}{l}\text { Patients analyzed } \\
\text { for primary } \\
\text { endpoint } \\
(N=1113)\end{array}$} & \multicolumn{3}{|c|}{ Sub-groups based on $\mathrm{Hb}$ levels at initiation of epoetin beta } & \multirow[t]{2}{*}{$P$ value } \\
\hline & & & $\begin{array}{l}\text { Group I } \\
10 \leq \mathrm{Hb}<11 \\
\mathrm{~g} / \mathrm{dL}(N=309)\end{array}$ & $\begin{array}{l}\text { Group II } \\
9 \leq \mathrm{Hb}<10 \\
\mathrm{~g} / \mathrm{dL}(N=545)\end{array}$ & $\begin{array}{l}\text { Group III } \\
\mathrm{Hb}<9 \mathrm{~g} / \mathrm{dL} \\
(N=259)\end{array}$ & \\
\hline $\begin{array}{l}\text { Systolic blood pressure } \\
(\mathrm{mmHg})^{\mathrm{a}}\end{array}$ & $\begin{array}{r}136.3 \pm 20.0 \\
(N=1331)\end{array}$ & $\begin{array}{c}136.2 \pm 19.7 \\
(N=924)\end{array}$ & $\begin{array}{c}138.1 \pm 19.6 \\
(N=262)\end{array}$ & $\begin{array}{c}134.5 \pm 19.5 \\
(N=445)\end{array}$ & $\begin{array}{l}137.2 \pm 20.1 \\
(N=217)\end{array}$ & 0.045 \\
\hline $\begin{array}{l}\text { Diastolic blood pressure } \\
(\mathrm{mmHg})^{\mathrm{a}}\end{array}$ & $\begin{array}{r}72.1 \pm 13.0 \\
(N=1325)\end{array}$ & $\begin{array}{r}72.1 \pm 13.0 \\
(N=920)\end{array}$ & $\begin{array}{r}75.1 \pm 13.6 \\
(N=262)\end{array}$ & $\begin{array}{r}71.2 \pm 12.3 \\
(N=443)\end{array}$ & $\begin{array}{r}70.1 \pm 13.0 \\
(N=215)\end{array}$ & $<0.0001$ \\
\hline Total protein $(\mathrm{g} / \mathrm{dL})^{\mathrm{a}}$ & $\begin{array}{l}6.6 \pm 0.7 \\
\quad(N=1354)\end{array}$ & $\begin{array}{l}6.6 \pm 0.7 \\
\quad(N=914)\end{array}$ & $\begin{array}{l}6.8 \pm 0.6 \\
\quad(N=255)\end{array}$ & $\begin{array}{l}6.6 \pm 0.8 \\
\quad(N=453)\end{array}$ & $\begin{array}{l}6.4 \pm 0.8 \\
\quad(N=206)\end{array}$ & $<0.0001$ \\
\hline Ferritin $(\mathrm{ng} / \mathrm{mL})^{\mathrm{a}}$ & $\begin{array}{c}185.1 \pm 183.4 \\
(N=710)\end{array}$ & $\begin{array}{c}176.3 \pm 181.0 \\
(N=457)\end{array}$ & $\begin{array}{c}160.1 \pm 139.3 \\
(N=137)\end{array}$ & $\begin{array}{c}165.7 \pm 140.6 \\
(N=220)\end{array}$ & $\begin{array}{c}222.0 \pm 279.0 \\
(N=100)\end{array}$ & 0.016 \\
\hline $\operatorname{TSAT}(\%)^{\mathrm{a}}$ & $\begin{array}{r}27.8 \pm 11.5 \\
(N=680)\end{array}$ & $\begin{array}{r}28.6 \pm 10.6 \\
(N=436)\end{array}$ & $\begin{array}{r}29.9 \pm 10.7 \\
(N=138)\end{array}$ & $\begin{array}{r}28.9 \pm 10.7 \\
(N=203)\end{array}$ & $\begin{array}{c}26.3 \pm 10.2 \\
(N=95)\end{array}$ & 0.037 \\
\hline Albumin $(\mathrm{g} / \mathrm{dL})^{\mathrm{a}}$ & $\begin{array}{l}3.6 \pm 0.6 \\
\quad(N=1431)\end{array}$ & $\begin{array}{l}3.6 \pm 0.6 \\
\quad(N=973)\end{array}$ & $\begin{array}{l}3.8 \pm 0.5 \\
\quad(N=268)\end{array}$ & $\begin{array}{l}3.7 \pm 0.5 \\
\quad(N=480)\end{array}$ & $\begin{array}{l}3.4 \pm 0.6 \\
\quad(N=225)\end{array}$ & $<0.0001$ \\
\hline Calcium $(\mathrm{mg} / \mathrm{dL})^{\mathrm{a}}$ & $\begin{array}{l}8.7 \pm 0.7 \\
\quad(N=1341)\end{array}$ & $\begin{array}{l}8.7 \pm 0.7 \\
\quad(N=908)\end{array}$ & $\begin{array}{l}8.9 \pm 0.6 \\
(N=250)\end{array}$ & $\begin{array}{l}8.7 \pm 0.6 \\
\quad(N=445)\end{array}$ & $\begin{array}{l}8.5 \pm 0.7 \\
(N=213)\end{array}$ & $<0.0001$ \\
\hline Phosphorus $(\mathrm{mg} / \mathrm{dL})^{\mathrm{a}}$ & $\begin{array}{l}3.9 \pm 0.8 \\
\quad(N=1237)\end{array}$ & $\begin{array}{l}3.9 \pm 0.8 \\
\quad(N=833)\end{array}$ & $\begin{array}{l}3.8 \pm 0.8 \\
\quad(N=222)\end{array}$ & $\begin{array}{l}3.8 \pm 0.7 \\
\quad(N=409)\end{array}$ & $\begin{array}{l}4.1 \pm 0.9 \\
\quad(N=202)\end{array}$ & 0.0001 \\
\hline $\begin{array}{l}\text { Total cholesterol (mg/ } \\
\mathrm{dL})^{\mathrm{a}}\end{array}$ & $\begin{array}{r}175.7 \pm 40.9 \\
(N=1056)\end{array}$ & $\begin{array}{c}174.6 \pm 38.9 \\
(N=704)\end{array}$ & $\begin{array}{c}178.0 \pm 38.0 \\
(N=201)\end{array}$ & $\begin{array}{l}175.1 \pm 40.4 \\
(N=341)\end{array}$ & $\begin{array}{c}169.5 \pm 36.4 \\
(N=162)\end{array}$ & 0.11 \\
\hline $\begin{array}{l}\text { C-reactive protein }(\mathrm{mg} / \\
\mathrm{dL})^{\mathrm{a}}\end{array}$ & $\begin{array}{l}0.5 \pm 1.5 \\
\quad(N=915)\end{array}$ & $\begin{array}{l}0.5 \pm 1.6 \\
\quad(N=583)\end{array}$ & $\begin{array}{l}0.4 \pm 1.1 \\
\quad(N=139)\end{array}$ & $\begin{array}{l}0.3 \pm 0.6 \\
\quad(N=287)\end{array}$ & $\begin{array}{l}1.0 \pm 2.7 \\
\quad(N=157)\end{array}$ & $<0.0001$ \\
\hline $\begin{array}{l}\text { Urine protein/ } \\
\text { creatinine }^{\mathrm{a}}\end{array}$ & $\begin{array}{l}2.8 \pm 5.2 \\
\quad(N=716)\end{array}$ & $\begin{array}{l}2.9 \pm 5.9 \\
\quad(N=480)\end{array}$ & $\begin{array}{l}3.2 \pm 9.0 \\
\quad(N=133)\end{array}$ & $\begin{array}{l}2.4 \pm 3.9 \\
\quad(N=240)\end{array}$ & $\begin{array}{l}3.5 \pm 4.6 \\
\quad(N=107)\end{array}$ & 0.22 \\
\hline $\begin{array}{l}\text { Starting dose of epoetin } \\
\text { beta }(\mathrm{IU} / \mathrm{month})^{\mathrm{a}}\end{array}$ & $\begin{array}{l}12806.4 \pm 6792.4 \\
(N=1436)\end{array}$ & $\begin{array}{l}12135.8 \pm 6757.1 \\
\quad(N=972)\end{array}$ & $\begin{array}{l}11123.1 \pm 6085.6 \\
(N=260)\end{array}$ & $\begin{array}{l}11758.0 \pm 6608.9 \\
(N=471)\end{array}$ & $\begin{array}{l}13966.8 \pm 7380.1 \\
(N=241)\end{array}$ & $<0.0001$ \\
\hline
\end{tabular}

$N$ number of patients, $S D$ standard deviation, $C K D$ chronic kidney disease, TSAT transferrin saturation

${ }^{\text {a }}$ Values were mean $\pm \mathrm{SD}$

Table 2 Lead time and changes of laboratory test values

\begin{tabular}{lccc}
\hline Variables & $\begin{array}{l}\text { Group I } \\
10 \leq \mathrm{Hb}<11 \mathrm{~g} / \mathrm{dL}(N=309)\end{array}$ & $\begin{array}{l}\text { Group II } \\
9 \leq \mathrm{Hb}<10 \mathrm{~g} / \mathrm{dL}(N=545)\end{array}$ & $\begin{array}{l}\text { Group III } \\
\mathrm{Hb}<9 \mathrm{~g} / \mathrm{dL}(N=259)\end{array}$ \\
\hline When $\mathrm{Hb}$ levels decreased below $11 \mathrm{~g} / \mathrm{dL}$ for the first time & & $10.4 \pm 0.5$ \\
$\mathrm{Hb}(\mathrm{g} / \mathrm{dL})^{\mathrm{a}}$ & $10.5 \pm 0.4$ & $10.5 \pm 0.5$ & $2.3 \pm 1.2$ \\
$\mathrm{sCr}(\mathrm{mg} / \mathrm{dL})^{\mathrm{a}}$ & $2.7 \pm 1.3$ & $2.4 \pm 1.2$ & $27.7 \pm 16.8$ \\
eGFR $\left(\mathrm{mL} / \mathrm{min} / 1.73 \mathrm{~m}^{2}\right)^{\mathrm{a}}$ & $23.6 \pm 12.3$ & $24.6 \pm 13.1$ & 0.027 \\
When epoetin beta therapy was initiated & $10.4 \pm 0.3$ & $9.5 \pm 0.3$ & $8.3 \pm 0.6$ \\
$\mathrm{Hb}(\mathrm{g} / \mathrm{dL})^{\mathrm{a}}$ & $2.9 \pm 1.4$ & $3.0 \pm 1.3$ & $3.4 \pm 1.5$ \\
$\mathrm{sCr}(\mathrm{mg} / \mathrm{dL})^{\mathrm{a}}$ & $21.4 \pm 11.0$ & $18.5 \pm 8.7$ & $16.8 \pm 8.1$
\end{tabular}

$\mathrm{Hb}$ hemoglobin, $\mathrm{sCr}$ serum creatinine, $e G F R$ estimated glomerular filtration rate

${ }^{\text {a }}$ Values were mean $\pm \mathrm{SD}$

comorbidities, and there was variation, for example, in the eGFR levels of each group when $\mathrm{Hb}$ levels decreased below $11 \mathrm{~g} / \mathrm{dL}$ (Table 2), analysis was performed after using the IPW method to adjust for selection bias, the second issue. As described, analysis was performed in this study on the basis of a study design that resolves the issues that would normally arise in an observational study of the appropriate timing for initiation of ESA therapy. 
Table 3 Adjusted hazard ratio for renal events

\begin{tabular}{|c|c|c|c|}
\hline & Hazard ratio & $95 \% \mathrm{CI}$ & $P$ value \\
\hline \multicolumn{4}{|c|}{ (A) Hazard ratio after adjusting for data of patients with weight above 99th percentile in Group II } \\
\hline Group I & Reference & & \\
\hline Group II & 1.48 & $0.91-2.40$ & 0.11 \\
\hline Group III & 2.52 & $1.98-3.21$ & $<0.0001$ \\
\hline \multicolumn{4}{|c|}{ (B) Hazard ratio after adjusting for data of patients with weight above 98th percentile in Group II } \\
\hline Group I & Reference & & \\
\hline Group II & 1.29 & $1.02-1.64$ & 0.033 \\
\hline
\end{tabular}

For weight calculation, the time-dependent covariates included in the model are sCr levels and Hb levels. We also included the following baseline covariates: age, sex, hypertension, heart failure, angina, arrhythmia, diabetes, hyperlipidemia, and hyperuricemia

In the comparison between Groups I and II, there were subjects with extreme weights (median 0.84, 98th percentile 3.74, 99th percentile 34.23, maximum 236,242.07). To account for these extreme weights, we compared the groups using weights truncated at the 99th percentile and 98th percentile

CI confidence interval

Table 4 Adjusted hazard ratio for cardiovascular events

\begin{tabular}{|c|c|c|c|}
\hline & Hazard ratio & $95 \% \mathrm{CI}$ & $P$ value \\
\hline \multicolumn{4}{|c|}{$\begin{array}{l}\text { Hazard ratio after adjusting for data of patients with weight abov } \\
\text { 98th percentile in Group II }\end{array}$} \\
\hline Group I & Reference & & \\
\hline Group II & 1.00 & $0.58-1.71$ & 0.99 \\
\hline Group III & 1.94 & $0.96-3.94$ & 0.066 \\
\hline
\end{tabular}

For weight calculation, the time-dependent covariates included in the model are $\mathrm{sCr}$ levels and $\mathrm{Hb}$ levels. We also included the following baseline covariates: age, sex, hypertension, heart failure, angina, arrhythmia, diabetes, hyperlipidemia and hyperuricemia. In the comparison between Groups I and II, there were subjects with extreme weights (median 1.03, 98th percentile 6.18 , 99th percentile 96.29 , maximum 495,481.07). To account for these extreme weights, we compared the groups using weights truncated at the 98th percentile

CI confidence interval

In analysis of the effects of renal events using the IPW method, to confirm that a comparison of Groups I and II would not change interpretation of the results, both a 99th percentile weight and a 98th percentile weight were used, resulting in the respective hazard ratios $1.48(95 \% \mathrm{CI}$, $0.91-2.40 ; P=0.11)$ and 1.29 (95\% CI, 1.02-1.64; $P=0.033)$. It is known that as weights are progressively truncated, the precision of the estimate increases, resulting in induced bias [13]. Using a 99th percentile weight would provide results closer to the true value but with a wider confidence interval than when using a 98th percentile weight. Generally speaking, even if the hazard ratio is close to 1 , a narrow confidence interval could show a significant difference, and although such a difference would be statistically significant, it would have little clinical value (e.g., HR, 1.05; 95 \% CI, 1.01-1.09). On the other hand, even without a statistically significant difference, its effect could be sufficiently suggested by a hazard ratio further from 1 if the confidence interval is kept somewhat narrow. The results this time with the 99th percentile weight are similar.

By using the above study design and analysis method, this study demonstrated that initiation of ESA therapy when $\mathrm{Hb}$ levels decreased below $11 \mathrm{~g} / \mathrm{dL}$ could reduce the risk of renal events in non-dialysis CKD patients with anemia more effectively than initiation of ESA therapy at below $9 \mathrm{~g} / \mathrm{dL}$. Also, sensitivity analysis showed that initiation of ESA therapy when $\mathrm{Hb}$ levels decreased below $11 \mathrm{~g} / \mathrm{dL}$ could even reduce the risk of renal events more effectively than initiation of ESA therapy at below $10 \mathrm{~g} /$ $\mathrm{dL}$.

Starting in 2000, Gouva et al. studied 88 nondiabetic patients in Greece to compare renal prognosis between a group that started ESA treatment early and a group that deferred ESA treatment. They found that renal prognosis in the early ESA treatment group was significantly better [8], which is similar to the findings in the present study.

This study supports the recommendation in the 2008 guideline for renal anemia in CKD patients presented by the Japanese Society for Dialysis Therapy, which states that ESA therapy should be started when the $\mathrm{Hb}$ level is less than $11 \mathrm{~g} / \mathrm{dL}$ in non-dialysis patients [14]. It is necessary to consider each patient's condition when deciding the appropriate time to start treatment, as recommended in the 2013 Evidence-based Clinical Practice Guideline for CKD published by the Japanese Society of Nephrology [15].

This study has some limitations. Because this is an observational study, no randomization was used in comparison of Japanese patients based on the $\mathrm{Hb}$ levels at initiation of epoetin beta. The timing of epoetin beta 


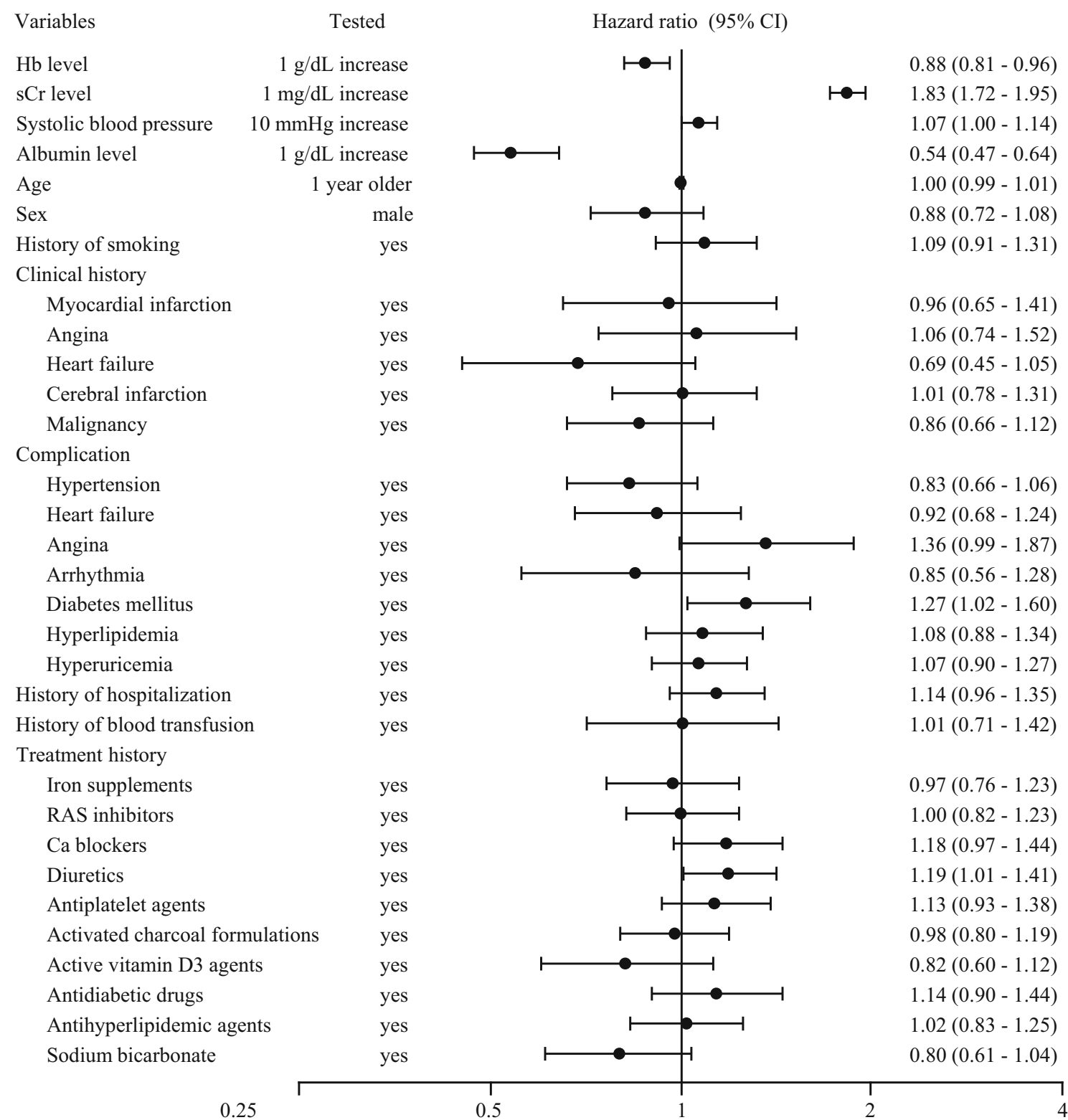

Fig. 3 Prognosis factors for renal survival. $\mathrm{Hb}$ hemoglobin, $s \mathrm{Cr}$ serum creatinine, $R A S$ renin-angiotensin system, $C a$ calcium, $C I$ confidence interval. The Cox regression model was used to assess relationships between patient baseline characteristics (including $\mathrm{Hb}$ levels, sCr levels, age, sex, comorbidities, and medical history;

initiation depended on each nephrologist evaluating the patient's total physical condition, and subjective bias might have affected the results of this study. Thus, the findings should be interpreted with this in mind, although the IPW method was used to adjust for artificial censoring before calculating hazard ratios for the primary endpoint. To account for lead-time bias, the date, $\mathrm{Hb}$ level, and $\mathrm{sCr}$ level at the time $\mathrm{Hb}$ levels decreased below $11 \mathrm{~g} / \mathrm{dL}$ were retrospectively collected, but data comorbidities and medical histories observed in more than $5 \%$ of patients in each group and with less than $20 \%$ of data missing were included) and outcomes for those patients eligible for efficacy analysis

on albumin and urinary protein were not. Therefore, analysis of renal events using the IPW method could not be adjusted for albumin or urinary protein values. Also, we were unable to capture patients with events occurring before treatment initiation, which could bias results in favor of the deferred treatment initiation group. Response and resistance to ESAs were also not evaluated in this study, and missing data might have affected the results. 
Table 5 Adverse reactions (number of patients)

\begin{tabular}{|c|c|c|c|c|c|c|}
\hline \multirow{2}{*}{$\begin{array}{l}\text { Patients analyzed for } \\
\text { safety }\end{array}$} & \multicolumn{5}{|c|}{$\mathrm{Hb}$ levels at initiation of epoetin beta } & \multirow{2}{*}{$\begin{array}{l}\text { Total } \\
(N=1714)\end{array}$} \\
\hline & $\begin{array}{l}11 \mathrm{~g} / \mathrm{dL} \leq \mathrm{Hb} \\
(N=45)\end{array}$ & $\begin{array}{l}10 \leq \mathrm{Hb}<11 \mathrm{~g} / \mathrm{dL} \\
(N=352)\end{array}$ & $\begin{array}{l}9 \leq \mathrm{Hb}<10 \mathrm{~g} / \mathrm{dL} \\
(N=757)\end{array}$ & $\begin{array}{l}\mathrm{Hb}<9 \mathrm{~g} / \mathrm{dL} \\
(N=521)\end{array}$ & $\begin{array}{l}\text { Unknown } \\
(N=39)\end{array}$ & \\
\hline Thrombocytopenia & & $1(0.3 \%)$ & & & & $1(0.1 \%)$ \\
\hline $\begin{array}{l}\text { Cerebral } \\
\text { hemorrhage }^{\mathrm{a}}\end{array}$ & & & $2(0.3 \%)$ & & & $2(0.1 \%)$ \\
\hline Cerebral infarction ${ }^{\mathrm{a}}$ & & $1(0.3 \%)$ & & & & $1(0.1 \%)$ \\
\hline $\begin{array}{l}\text { Acute myocardial } \\
\text { infarction }^{\mathrm{a}}\end{array}$ & & & & $1(0.2 \%)$ & & $1(0.1 \%)$ \\
\hline $\begin{array}{l}\text { Aortic aneurysm } \\
\text { rupture }^{\mathrm{a}}\end{array}$ & & & & $1(0.2 \%)$ & & $1(0.1 \%)$ \\
\hline Hypertension & & & $1(0.1 \%)$ & $1(0.2 \%)$ & & $2(0.1 \%)$ \\
\hline Pruritus & & & $1(0.1 \%)$ & $1(0.2 \%)$ & & $2(0.1 \%)$ \\
\hline Chest discomfort & & & $1(0.1 \%)$ & & & $1(0.1 \%)$ \\
\hline Injection site pain & & $1(0.3 \%)$ & & & & $1(0.1 \%)$ \\
\hline $\begin{array}{l}\text { Blood pressure } \\
\text { increased }\end{array}$ & & & $1(0.1 \%)$ & & & $1(0.1 \%)$ \\
\hline Total & $0(0.0 \%)$ & $3(0.9 \%)$ & $6(0.8 \%)$ & $4(0.8 \%)$ & $0(0.0 \%)$ & $13(0.8 \%)$ \\
\hline
\end{tabular}

Incidence of adverse reactions was tabulated for the 1714 patients in the safety analysis set

a Serious adverse reactions

\section{Conclusion}

Initiation of ESA therapy when $\mathrm{Hb}$ levels decreased below $11 \mathrm{~g} / \mathrm{dL}$ but not below $10 \mathrm{~g} / \mathrm{dL}$ could be more effective at reducing the risk of renal events in non-dialysis CKD patients with anemia compared with initiation of ESA therapy at below $9 \mathrm{~g} / \mathrm{dL}$ or even $10 \mathrm{~g} / \mathrm{dL}$.

Acknowledgments The authors would like to express our deepest gratitude to the patients, in addition to the investigators and staff at the study sites (presented in this section) for their contribution to the study. This study was sponsored by Chugai Pharmaceutical, Co., Ltd. (Tokyo, Japan). The JET-STREAM study group comprised the following 346 hospitals or clinics in Japan: Hokkaido: Sapporo Medical University Hospital; Sapporo Hokuyu Hospital; Tenshi Hospital Medical Corporation Bokoi; KKR Sapporo Medical Center Tonan Hospital; NHO Hokkaido Medical Center; Iwamizawa Municipal General Hospital; Monbetsu National Health Insurance Hospital; Tomakomai Nisshou Hospital; Nikko Memorial Hospital; Kushiro City General Hospital; Obihiro Kosei General Hospital; Goryoukaku Nephroclinic; Hakushoukai-Sano Hospital. Aomori: Hakuseikai Icho Hospital; Oyokyo Kidney Research Institute; Hachinohe Heiwa Hospital; Hachinohe West Hospital; Ooma Hospital. Iwate: San-ai Hospital, Yahaba Clinic in affiliation with San-ai Hospital. Miyagi: Tohoku Kosai Miyagino Hospital; JCHO Sendai Hospital; Koujinkai Chyu-o Clinic; Koujinkai Kimachi Hospital; Senseki Hospital. Yamagata: Tsuruoka Municipal Shonai Hospital. Fukushima: Ohara General Hospital; Iwaki Urology Clinic; Onoda Hospital. Ibaragi: Hitachi General Hospital; Nagase Medical Clinic; University of Tsukuba Hospital; Tsukuba Gakuen Hospital; Tokyo Medical University Ibaraki Medical Center. Tochigi: Takemura Medical Nephro Clinic; Kohira Medical Clinic; Hashimoto Kidney Clinic. Gunma: Saiseikai Maebashi Hospital; Maebashi Hirosegawa Clinic; SARUKI CLINIC, Shibukawa Central Hospital; Yamada Clinic;
Kusunoki Hospital; Toho Hospital; NHO Takasaki General Medical Center. Saitama: JCHO Saitama Medical Center; Saitamatsukuba Hospital; Saiseikai Kawaguchi General Hospital; Koshigaya Municipal Hospital; Misato Kenwa Clinic; Saitama Tsukinomori Clinic; Saitama Ken-oh Hospital; Kitasato University Medical Center; Saitama Medical University; Musashiranzan Hospital; Fukaya Red Cross Hospital; Kita-asaka Ekimae Clinic; Saitama Medical Center, Saitama Medical University; Sakura Memorial Hospital; NHO Nishisaitamachuo Hospital; Sekishindo Hospital. Chiba: Kameda Medical Center; Nippon Medical School Chiba Hokusoh Hospital; Tokyo Dental College Ichikawa General Hospital; Juntendo University Urayasu Hospital; Tokatsu Clinic Hospital. Tokyo: Saiseikai Central Hospital; The Juntendo University Hospital; The Jikei University School of Medicine; Medical Hospital of Tokyo Medical and Dental University; Toranomon Hospital; Toranomon Hospital Kajigaya; Tokyo Medical University; St. Luke's International Hospital; Tokyo Women's Medical University, Department of Medicine, Kidney Center, and Division of Nephrology and Hypertension, Diabetes Center; Nippon Medical School Hospital; Kisenkai Kisen Hospital; Ikegami General Hospital; Ogawa Clinic; NTT Medical Center Tokyo; Nissan Tamagawa Hospital; Toho University Ohashi Medical Center; Kanto Central Hospital; Kidney Clinic Setagaya; Nakano General Hospital; Itabashi Hospital, Nihon University School of Medicine; Akabane Central General Hospital; Tokyo Metropolitan Ohtsuka Hospital; National Disaster Medical Center; Kyorin University Hospital; Musashino Red Cross Hospital; Tokyo Metropolitan Tama Medical Center. Kanagawa: Seirei Yokohama Hospital; H.E.C. Science Clinic; JCHO Yokohama Central Hospital; International Goodwill Hospital; Yokohama Municipal Citizen's Hospital; NHO Yokohama Medical Center; Tsurumi-Nishiguchi Hospital; Yokohama Yousai Hospital; Heiwa Hospital; Tachibanadai Hospital; Nagatsuda Kosei General Hospital; Kawasaki Saiwai Clinic; Asao Kidney Clinic; General Takatsu Central Hospital; Teikyo University School of Medicine University Hospital, Mizonokuchi; Kanto Rosai Hospital; Kawasaki Municipal Ida Hospital; Synthesis Shinkawabashi Hospital; Yokosuka Kyosai Hospital; 
Yokosuka City Hospital; Shonan Kamakura General Hospital; St. Joseph's Hospital; Yokosuka General Hospital Uwamachi; Yokohama City University Medical Center; Sagamihara Clinic; Hirose Hospital; Sohbudai Nieren Clinic; Ebina General Hospital; Sagami Junkanki Clinic; Tsuruma Ekichikajin Clinic; Toshiba Rinkan Hospital; Tokai University School of Medicine; Tokai University Oiso Hospital. Niigata: Saiseikai Niigata Dai-ni Hospital; Niigata University; Shinrakuen Hospital; Sado General Hospital; Toyosaka Hospital; Nanbugo Kosei Hospital; Kido Hospital; Sado General Hospital; Niigata Prefectural Yoshida Hospital; Joetsu General Hospital; Itoigawa General Hospital. Toyama: Toyama Rosai Hospital; Kamiichi General Hospital; Toyama City Hospital; Koseiren Takaoka Hospital. Ishikawa: Public Hakui Hospital; Ishikawa Prefectural Central Hospital; Noto General Hospital; Kanazawa Medical University; Keiju Medical Center; Division of Nephrology, and Division of Rheumatology, Department of Internal Medicine, Kanazawa University Hospital. Fukui: Fukui Prefectural Hospital; Municipal Tsuruga Hospital; Fukui Saiseikai Hospital; Hayashi Clinic. Yamanashi: Kanoiwa General Hospital; Yamanashi Kosei Hospital. Nagano: Iida Municipal Hospital; Shinshu University Hospital; Chikuma Central Hospital; Hokushin General Hospital; Maruko Central General Hospital; Saku General Hospital. Gifu: Tokai Central Hospital; Hirano General Hospital; Gihoku Kosei Hospital; Asahi University Murakami Memorial Hospital; Gifu University Hospital; Gifu Municipal Hospital; Ogaki Municipal Hospital; Tajimidaiichi Hospital; Tohno Kousei Hospital; Toki General Hospital; Chuno Kosei Hospital; Kizawa Memorial Hospital. Shizuoka: Chutoen General Medical Center; Hamamatsu Medical Center; Fujinomiya City General Hospital; Numazu City Hospital. Aichi: Kasugai Municipal Hospital; Chukyo Hospital; Nagoya Kyoritsu Hospital; Chubu Rosai Hospital; Kyoritsu General Hospital; Kainan Hospital; Aichi Medical University Hospital; Konan Kosei Hospital; Nagoya Memorial Hospital; Shinseikai Dai-ichi Hospital; Meijo Hospital. Mie: Japanese Red Cross Society Ise Hospital; Takeuchi Hospital; Mie University Hospital; Suzuka General Hospital; Kuwana Medical Center-East Branch; Yokkaichi Municipal Hospital. Shiga: Omihachiman Community Medical Center; Kusatsu General Hospital; Shiga University of Medical Science; JCHO Shiga Hospital. Kyoto: Mitsubishi Kyoto Hospital; Kyoto Daiichi Red Cross Hospital; NHO Kyoto Medical Center; Rakuwakai-Otowa Kinen Hospital; Rakuwakai-Otowa Hospital; Nishijin Hospital; Kyoto Miniren Chuo Hospital; Kyoto Prefectural University Hospital; Tango Central Hospital; Kyoto-Katsura Hospital. Osaka: Suita Municipal Hospital; Osaka University Hospital; Saiseikai Suita Hospital; Takatsuki General Hospital; Osaka Medical College Hospital; Osaka City University Graduate School of Medicine; Osaka National Hospital; Osaka General Medical Center; Minamiosaka Hospital; Shirasagi Hospital; Saiseikai Nakatsu Hospital; JCHO Osaka Hospital; Kitano Hospital; Chibune Kidney and Dialysis Clinic; Medical Corporation Kyoujinkai Komatsu Hospital; Kansai Medical University Takii Hospital; Matsushita Memorial Hospital; Fuchu Hospital; Rinku General Medical Center; Fujii Clinic; PL Hospital; Tanakakitanoda Hospital. Hyogo: Rokko Island Kohnan Hospital; Kohnan Hospital: Kobe City Medical Center General Hospital; Kobe University Graduate School of Medicine; Sumiyoshigawa Hospital; Hyogo Prefectural Awaji Medical Center; Meimai Central Hospital; Meiwa General Hospital; Kohnan Kakogawa Hospital. Nara: Tenri Hospital. Wakayama: Kasei Tamura Hospital; Ujita Clinic; Japanese Red Cross Wakayama Medical Center; Kinokawa Clinic; Kisen KD Clinic; Kodama Hospital; Sakuragaoka Hospital; Kinan Hospital. Tottori: Tottori University Hospital; San-in Rosai Hospital; Japanese Red Cross Tottori Hospital; Taniguchi Hospital. Shimane: Shimane University Hospital; Shimane Prefectural Central Hospital. Okayama: Shigei Hospital; NHO Okayama Medical Center; Okayama Saiseikai General Hospital; Satou Memorial Hospital; Minamigata Clinic. Hiroshima: Teraoka Memorial Hospital; JA Hiroshima General
Hospital; Chuoh Naika Clinic; Harada Hospital; Phoenix Clinic; NHO Hiroshimanishi Medical Center; Shobara Red Cross Hospital; Motonaga Hospital; Inokuchi Clinic; Hiroshima Red Cross Hospital and Atomic-bomb Survivors Hospital; Omachi Tsuchiya Clinic; Tsuchiya General Hospital; Onomichi Municipal Hospital; Onomichi Clinic. Yamaguchi: Shimonoseki City Hospital; JCHO Tokuyama Central Hospital. Tokushima: Tokushima Kensei Hospital; Kawashima Hospital; Oe Kyodo Hospital. Kagawa: Takinomiya General Hospital; Miyano Hospital; Mitoyo General Hospital; Kaisei Hospital; Kagawa University Hospital; Kagawa Rosai Hospital. Ehime: Matsuyama Shimin Hospital; Murakami Memorial Hospital; Housyasen Daiiti Hospital; Uwajima City Hospital; Shikoku Central Hospital. Kochi: Chikamori Hospital; Shimazu Hospital; Kochi Takasu Hospital. Fukuoka: Kyushu University Hospital; Hamanomachi Hospital; Japanese Red Cross Fukuoka Hospital; Fukuoka University Hospital; Saiseikai Futsukaichi Hospital; Murakami Karindoh Hospital; Shin Koga Hospital; Omuta City Hospital; Kurume Daiichi Hospital; Steel Memorial Yawata Hospital; Nakama Municipal Hospital; Kokura Memorial Hospital; Aso-Iizuka Hospital; Moji Ekisaikai Hospital. Saga: Saga-ken Medical Centre Koseikan; Saga University. Nagasaki: Nagasaki University Hospital, Second Department of Internal Medicine, and Division of Blood Purification; Shinzato Clinic; Sasebo City General Hospital; Isahaya Health Insurance General Hospital; Hokusho Central Hospital. Kumamoto: Kumamoto Chuo Hospital; Kumamoto General Hospital; Kikuchi Medical Association Hospital; Tsutsumi Hospital. Oita: Matsuyama Clinic Oita Nephro Internal Medicine Department; Oita Nakamura Hospital; Murakami Memorial Hospital. Miyazaki: Ikei Hospital; University of Miyazaki Hospital; Chiyoda Hospital; Miyazaki Prefectural Miyazaki Hospital; Fujimoto General Hospital. Kagoshima: Ikeda Hospital; Kagoshima Aishinkai Oosumikanoya Hospital. Okinawa: Tomishiro Central Hospital; Okinawa Nanbu Medical Center/ Okinawa Nanbu Children's Medical Center; Taiyohno Clinic.

\section{Compliance with ethical standards}

Conflict of interest Employment/Leadership position/Advisory role: TA (Kyowa Hakko Kirin, Japan Tobacco, Astellas, Nipro), YO (Chugai), MK (Chugai), SK (Chugai). Stock ownership or options: YO (Statocom). Honoraria: TA (Chugai, Bayer, Kyowa Hakko Kirin), YT (Chugai, Kyowa Hakko Kirin, Mitsubishi Tanabe, Torii), HHi (Chugai, Kyowa Hakko Kirin, Torii, Bayer, FUSO, Astellas), HHa (Chugai, Astellas, Daiichi-Sankyo), SN (Chugai, Kyowa Hakko Kirin, Novartis, Mitsubishi Tanabe), YO (Sanofi). Manuscript fees: TA (Astellas, Kyowa Hakko Kirin), HHi (Chugai, Kyowa Hakko Kirin, Torii, Bayer, FUSO, Astellas). Research funding: YT (Bayer, Asahi Kasei, Otsuka, Eisai), HHa (Chugai, Kyowa Hakko Kirin). Subsidies or Donations: SN (Chugai, Kyowa Hakko Kirin, Astellas, Takeda, Bayer). Endowed departments by commercial entities: YT (Chugai, Baxter). Travel fees, gifts, and others: YO (Yakult, Takeda).

Open Access This article is distributed under the terms of the Creative Commons Attribution 4.0 International License (http://crea tivecommons.org/licenses/by/4.0/), which permits unrestricted use, distribution, and reproduction in any medium, provided you give appropriate credit to the original author(s) and the source, provide a link to the Creative Commons license, and indicate if changes were made.

\section{References}

1. Erslev AJ, Besarab A. Erythropoietin in the pathogenesis and treatment of the anemia of chronic renal failure. Kidney Int. 1997;51:622-30. 
2. Silverberg D, Wexler D, Blum M, Wollman Y, Iaina A. The cardio-renal anaemia syndrome: does it exist? Nephrol Dial Transplant. 2003;18(suppl 8):viii7-12.

3. Kuriyama S, Tomonari H, Yoshida H, Hashimoto T, Kawaguchi Y, Sakai O. Reversal of anemia by erythropoietin therapy retards the progression chronic renal failure, especially in nondiabetic patients. Nephron. 1997;77:176-85.

4. Leaf DE, Goldfarb DS. Interpretation and review of health-related quality of life data in CKD patients receiving treatment for anemia. Kidney Int. 2009;75:15-24.

5. Singh AK, Szczech L, Tang KL, Barnhart H, Sapp S, Wolfson M, et al. Correction of anemia with epoetin alfa in chronic kidney disease. N Engl J Med. 2006;355:2085-98.

6. Pfeffer MA, Burdmann EA, Chen CY, Cooper ME, de Zeeuw D, Eckardt KU, et al. A trial of darbepoetin alfa in type 2 diabetes and chronic kidney disease. N Engl J Med. 2009;361:2019-32.

7. Kidney Disease: Improving Global Outcomes (KDIGO) CKD Work Group. KDIGO 2012 clinical practice guideline for anemia in chronic kidney disease. Kidney Int. 2012;2(suppl):279-335.

8. Gouva C, Nikolopoulos P, Ioannidis JP, Siamopoulos KC. Treating anemia early in renal failure patients slows the decline of renal function: a randomized controlled trial. Kidney Int. 2004;66:753-60.
9. Matsuo S, Imai E, Horio M, Yasuda Y, Tomita K, Nitta K, et al. Revised equations for estimated GFR from serum creatinine in Japan. Am J Kidney Dis. 2009;53:982-92.

10. Epogin [package insert]. Chugai Pharmaceutical Co., Ltd. Tokyo, Japan; 2014. http://www.e-search.ne.jp/ jpr/PDF/CHUGAI17. PDF. Accessed 10 June 2015.

11. Hernán MA, Lanoy E, Costagliola D, Robins JM. Comparison of dynamic treatment regimes via inverse probability weighting. Basic Clin Pharmacol Toxicol. 2006;98:237-42.

12. Robins JM, Hernan MA, Brumback B. Marginal structural models and causal inference in epidemiology. Epidemiology. 2000;11:550-60.

13. Cole SR, Hernan MA. Constructing inverse probability weights for marginal structural models. Am $\mathrm{J}$ Epidemiol. 2008;168:656-64.

14. Tsubakihara Y, Nishi S, Akiba T, Hirakata H, Iseki K, Kubota M, et al. 2008 Japanese Society for Dialysis Therapy: guidelines for renal anemia in chronic kidney disease. Ther Apher Dial. 2010;14:240-75.

15. Kimura K, Okada K, Imai Y, Tamura K, Nishi S, Wada T, et al. Japanese Society of Nephrology: evidence-based clinical practice guideline for CKD 2013. p. 63-72 (in Japanese). 